\title{
Three-dimensional construction of micrometer level in rat stomach by synchrotron radiation
}

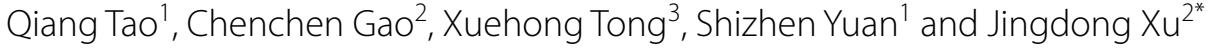

\author{
${ }^{*}$ Correspondence: \\ xujingdong@163.com \\ ${ }^{2}$ Department of Physiology \\ and Pathophysiology, \\ School of Basic Medicine, \\ Capital Medical University, \\ Beijing 100069, China \\ Full list of author information \\ is available at the end of the \\ article
}

\begin{abstract}
Background: The structural changes of gastric mucosa are considered as an important window of early gastric lesions. This article shows an imaging method of the stomach that does not use imaging agents. X-ray phase-contrast images of different stages of gastric development were taken using micrometer level X-ray in-line phase-contrast imaging (XILPCI) technique on synchrotron radiation facility. The aim of the study was to demonstrate that the imaging technique is an appropriate method for micron imaging of the gastric structures.

Methods: The stomachs of 4-, 6- and 12-week-old rats were removed and cleaned. XILPCI has 1000 times greater soft tissue contrast than that of X-ray traditional absorption radiography. The projection images of the rats stomachs were recorded by an XILPCI charge coupled device (CCD) at 9- $\mu \mathrm{m}$ image resolution.
\end{abstract}

Results: The X-ray in-line phase-contrast images of the different stages of rats' gastric specimens clearly showed the gastric architectures and the details of the gastroduodenal region. 3-dimensional (3D) stomach anatomical structure images were reconstruction.

Conclusion: The reconstructed gastric 3D images can clearly display the internal structure of the stomach. XILPCI may be a useful method for medical research in the future.

Keywords: Synchrotron radiation phase-contrast imaging, 3-dimensional gastric structure images, Different stages

\section{Background}

The stomach is an important organ in the alimentary tract. However, the imaging observation of its development has not been realized yet. This mainly employs gastroscopy which could obtain the image at a millimeter level. There are high-resolution images of $\mathrm{X}$-ray absorption of the human skeleton, but the images of the human abdominal organs remain poor. X-ray in-line phase-contrast imaging (XILPCI), using the X-ray phasechange after an X-ray passes through objects, has emerged as an imaging method and used to acquire micrometer level images of soft tissues.

(C) The Author(s) 2021. This article is licensed under a Creative Commons Attribution 4.0 International License, which permits use, sharing, adaptation, distribution and reproduction in any medium or format, as long as you give appropriate credit to the original author(s) and the source, provide a link to the Creative Commons licence, and indicate if changes were made. The images or other third party material in this article are included in the article's Creative Commons licence, unless indicated otherwise in a credit line to the material. If material is not included in the article's Creative Commons licence and your intended use is not permitted by statutory regulation or exceeds the permitted use, you will need to obtain permission directly from the copyright holder. To view a copy of this licence, visit http://creativecommons.org/ licenses/by/4.0/. The Creative Commons Public Domain Dedication waiver (http://creativecommons.org/publicdomain/zero/1.0/) applies to the data made available in this article, unless otherwise stated in a credit line to the data. 
XILPCI can be combined with computed tomography (CT) and form the phase-contrast $\mathrm{CT}$. It is also a kind of the diffraction $\mathrm{CT}$ and will probably be an imaging method for soft tissues without using imaging agents. The micrometer level image resolution of XILPCI of soft tissues can reach $0.37 \mu \mathrm{m}$ which make it able to obtain the more accuracy gastric structure. With the advantages mentioned above, this type of XILPCI method could be a good research method in the future of medicine.

A growing number of studies have shown that intestinal development has obvious temporal characteristics and is regulated by many factors [1]. The rapid growth and functional maturation of the stomach and small intestine in newborns may reflect an adaptation process, which include being exposed to an open environment and processing nutrient soon after the birth of newborns. Morphometrical analysis revealed that the growth rate is greater in the gastric corpus region than in the cardiac and pyloric regions, and the same increase is observed in the mucosal layer compared with the one in other layers. It has been shown that gastric mucosal cell proliferation was elevated during early postnatal development in the rat model [2]. Some evidence indicate that growth of gut mucosal tissues is associated with an enhanced DNA synthesis rate [3] and a decreased cell turnover rate in neonatal animals [4]. There are lots of researches on gastrointestinal cells, but few studies focus on the characteristic morphological in stomach and intestine of the rats. Our team focused on the XILPCI images of morphology and structure of normal rat stomach. Hence, we choose to use the young and adult rats as the objects of study.

\section{Results}

\section{Rats' gastric specimen characteristics in XILPCI projection images}

To intuitively understand the changes of gastric morphology and structure in different age groups, the XILPCI projection images of gastric specimens are shown in Fig. 1. Changes were observed at different week in rats. Figure 1A shows the ordered and regular tissues of a 4-week-old young gastric normal specimen, and the gastric walls are smooth without any hyperplasia. The XILPCI image are more detailed than the X-ray traditional image of a normal gastric specimen [5-8], as we could only see the overlapping walls of the stomach in the absorption images, and the images are very fuzzy with an unclear internal texture (as shown in Fig. 2).

On XILPCI images, a 4-week-old image shows a uniform grey level, which indicates that the wall of the 4-week-old gastric corpus is as thick as the gastric fundus. It is obvious that the wrinkles of the fundus are abundant, while the gastric fundus is much thinner. In the middle of Fig. 1B, C, there is a demarcation line between the gastric corpus and the gastric fundus. Figure 1B demonstrates the characteristics of a 6-week-old adult gastric normal specimen XILPCI image. The gastric wrinkles are more extensive, but the wrinkles of the 6-week-old gastric fundus are fewer than those of the 4-week-old gastric fundus, as shown in the image that the grey of a 6-week-old gastric fundus is lighter than that of a 4-week-old fundus. The 6-week-old gastric corpus is thicker than the 4-weekold gastric corpus for the grey of a 6-week-old gastric corpus is deeper in image than that of 4-week-old. Figure $1 \mathrm{C}$ demonstrates the characteristics of mature gastric normal tissues from a 12-week-old normal gastric specimen. The wrinkles in the walls of the gastric corpus and duodenum are the most abundant. 

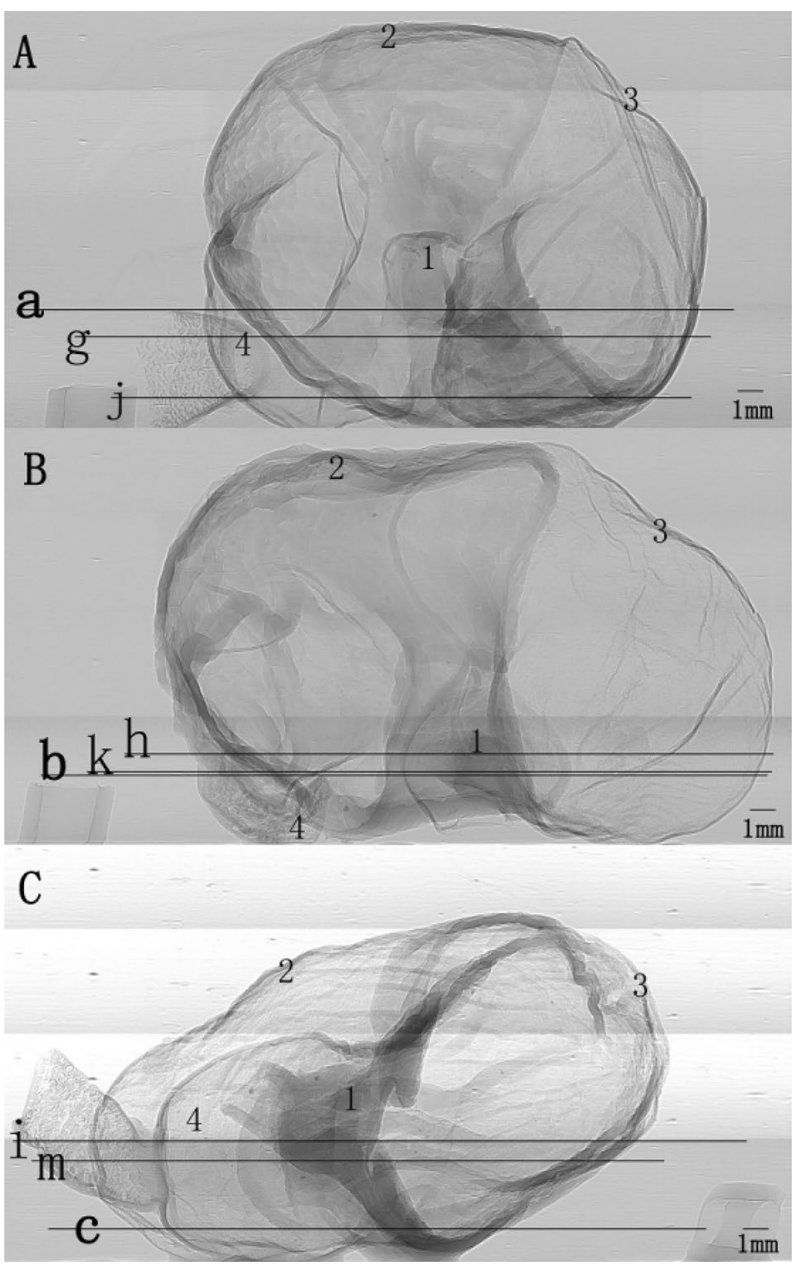

Fig. 1 XILPCI projection images of rats' gastric specimens. A A 4-week-old specimen showing a smooth gastric corpus wall. B A 6-week-old specimen showing the uneven gastric walls inside the stomach. C A 12-week-old specimen showing thick gastric corpus walls and more gastric corpus wrinkles. 1 Cardia, 2 Gastric Corpus, 3 Gastric Fundus, 4 Pylorus

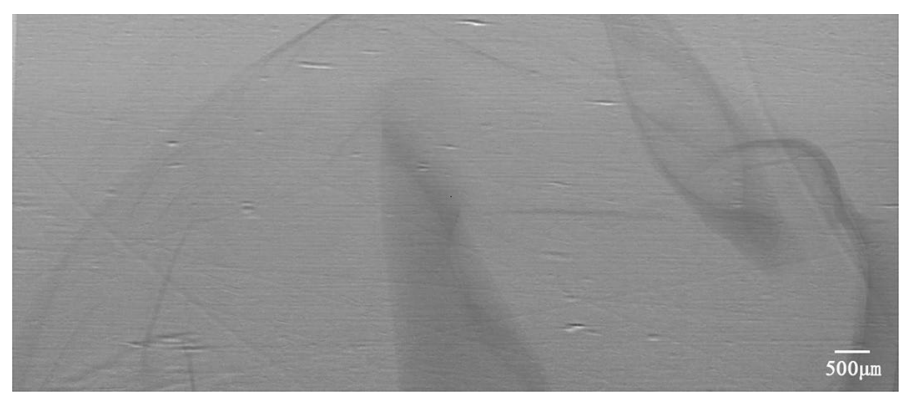

Fig. 2 An X-ray absorption image of a rat stomach 


\section{CT images of the same rats' gastric specimens}

The XILPCI 3-dimensional slices were rebuilt by means of a filter back projective algorithm. As shown in Fig. 3, the details can be visualized inside the gastric tissue from under the gross anatomy and the gastric inner surfaces clearly presented longitudinal branching wrinkle and gastric pits.

Traditional absorption CT images of a normal gastric specimen were obtained by SIEMENS Inveon Scanners with minimum resolution of $11 \mu \mathrm{m}$ and Inveon Acquisition Workplace with 1.5 Service Pack. The X-ray energy was $80 \mathrm{keV}$ and $400 \mu \mathrm{A}$. The absorption $\mathrm{CT}$ image of a gastric specimen was Fig. 4. However, it was not clearer than gastric phase-contrast images and the wall fold of the normal gastric specimen cannot be observed.

The d, e and f parts of the gastric specimens in Fig. 3 were taken out and fixed in formalin solution. Further analysis of these specimens show the same shape as Fig. 5, but XILPCI images could not show the clear internal structure the same as Fig. 5. At present, the gold standard for diagnosis is still biopsy. Hematoxylin-eosin (HE) staining

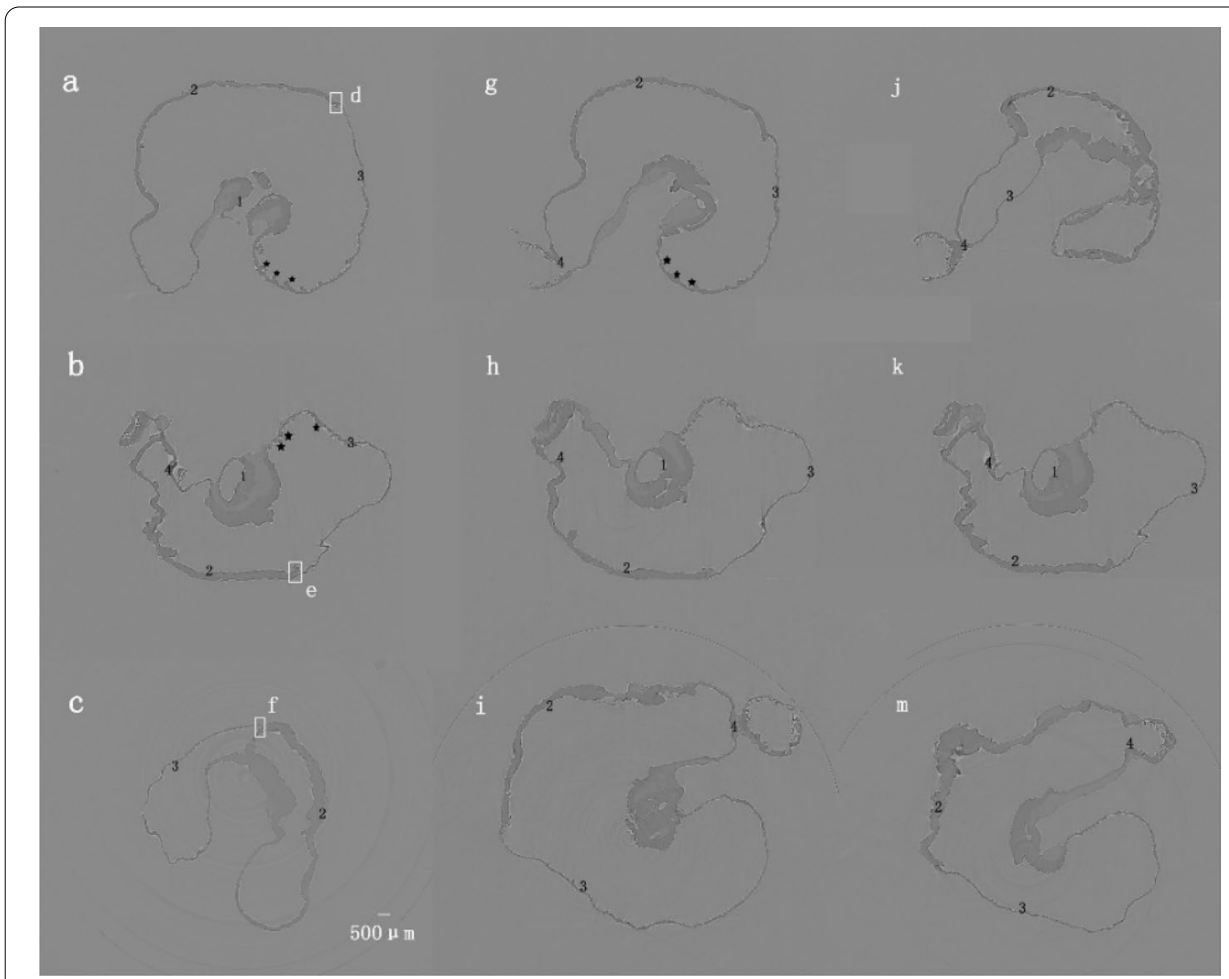

Fig. 3 The XILPCI transverse CT images of the rats' gastric specimens in Fig. 1. a The transverse images along the black line $\mathrm{a}, \mathrm{g}$ and $\mathrm{j}$ are shown in Fig. 1. There are transverse CT images of a 4-week-old rat stomach. It can clearly show that there are grey changes in different gastric structures. The thickness of the gastric fundus and corpus are the same as the XILPCI image shows equal width of gastric wall, and it is obvious that there are multiple bulges in the fundus of the 4-week-old rat stomach. $\mathbf{b}$ The transverse images along the black line $b, h$ and $k$ as shown in Fig. 1. The XILPCI images show the wider gastric corpus than the fundus but thicker walls of gastric corpus than that of the fundus. This obviously shows that there are grey changes in the different gastric structures and the duodenum. The bulges have decreased in the fundus of the 6-week-old rat stomach. c The transverse images along the black line $c$, $i$ and $m$ as shown in Fig. 1. It can obviously be seen that there are many gastric corpus wrinkles in the 12 -week-old gastric specimen. $\star \star \star$ represents the wrinkles of the gastric fundus. 1 Cardia, 2 Gastric Corpus, 3 Gastric Fundus, 4 Pylorus 


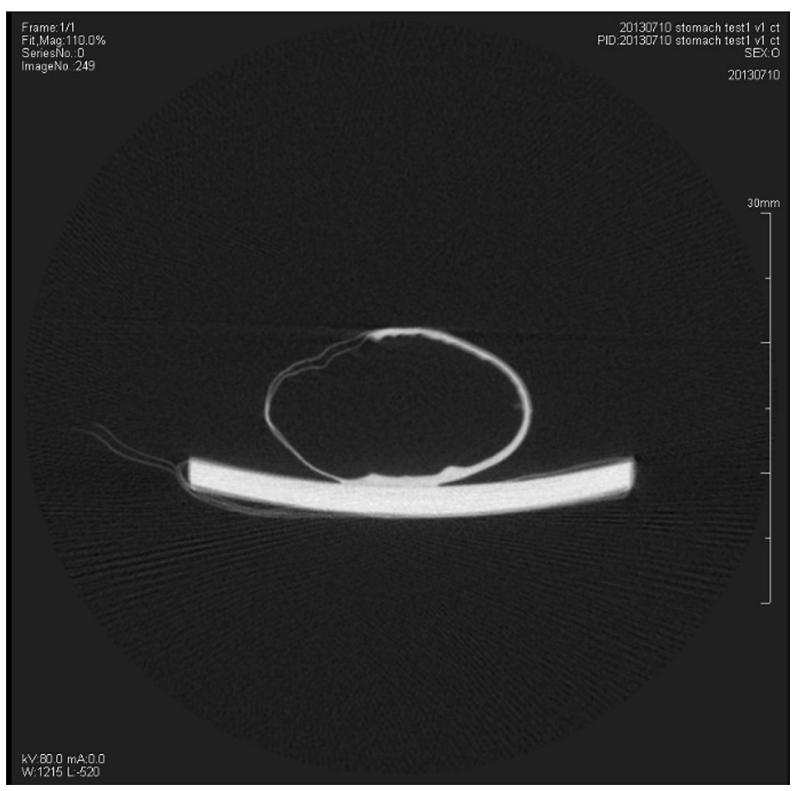

Fig. 4 The traditional absorption CT image of the normal rat's gastric specimen

processes are as follow: these gastric specimens were dehydrated and dried, and then the $\mathrm{d}$, e and $\mathrm{f}$ part of specimens were embedded in paraffin. Paraffin-embedded sections were made into pathological section with $5 \mu \mathrm{m}$ thickness and stained with HE to evaluate general morphology. In the body portion of the stomach after HE staining, the micrograph and macrograph showed a cross-section of the gastric wall (Fig. 5). Similar to the other parts of the gastrointestinal tract, there are four layers of structure in the gastric wall, which are outer mucosa, inner submucosa, muscular external layer, and serosa. During the growth and development of the rat, the fundus, formed by the upper curvature of the organ from the muscular external layer, is the thickest part at all three ages (Fig. $5 \mathrm{~d}-\mathrm{f}$ ). There is an obvious line between the gastric fundus and corpus. A mucosa layer is also palpable, but with overt differences seen on HE staining, and we could see that the mucosa layer in the 4-week-old rats show more alkalinity than that in the 6- and 12-week-old rats, especially at 12 weeks (Fig. 5f). The line could also be observed in Fig. $5 \mathrm{~d}-\mathrm{f}$ between the gastric fundus and corpus.

\section{Three-dimensional reconstruction images of the same rats' gastric specimens}

To further reconfirm the advantage of the XILPCI 3-dimensional images, we reconstructed 3-dimensional images of the different stages of the stomach as shown in Fig. 6. The XILPCI 3-dimensional images show a clear structure of the interior stomach (showed as Additional file 1: Video S1), and we could see the clear images of the villus of the fundus in the young rat stomach. There is a clear demarcation line between the gastric fundus and corpus. The wall of the 12-week-old rat stomach is thicker than those of the 4-week-old. The conclusion here shows that XILPCI has a high anatomical accuracy for stomachs images. In addition, the thickness of the gastric wall at various stages was measured in Fig. 6 to compare the thickness of gastric specimens. As shown in Fig. 7, the results indicate that the average value of 

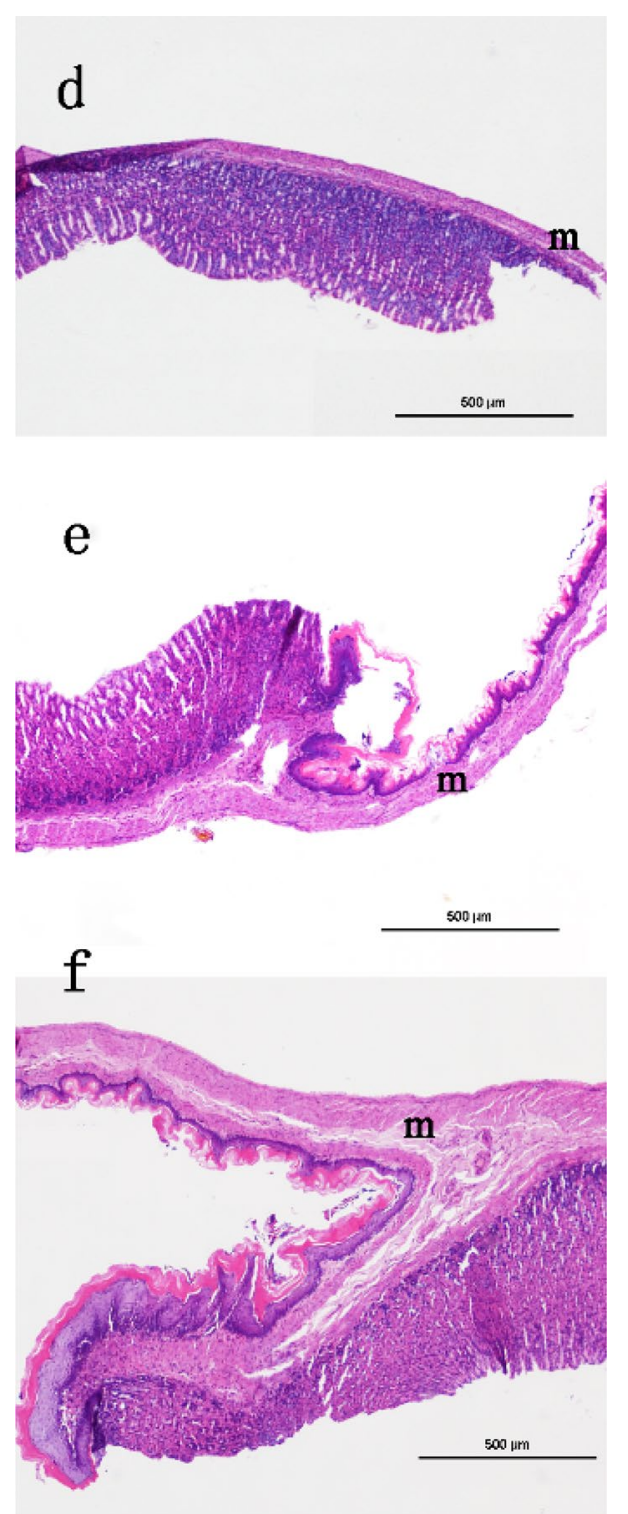

Fig. 5 Rats' gastric HE staining images. $\mathbf{d}$ A 4-week-old gastric specimen; e a 6-week-old specimen; $\mathbf{f}$ a 12-week-old gastric specimen

gastric wall at the same stage is very similar, and the measurement of gastric wall by 3-dimensional images in Fig. 6 has certain reference significance. Just as shown in Fig. 7a, the thickness of 4-week-old gastric wall for the right panel is lower than that of the left one, but the thickness of 6-week-old gastric wall is higher for the right panel, probably because of the randomness of specimen selection and the existence of measurement error. To further confirm the experimental evidence, we measured the thickness of gastric wall of the dried specimens which can be directly used for XILPCI experiments. As the Fig. 7b shown, it is clearly indicated the different types of gastric specimens shrink proportionally. However, these results are also in line with the requirements of statistics and within a reasonable range. 


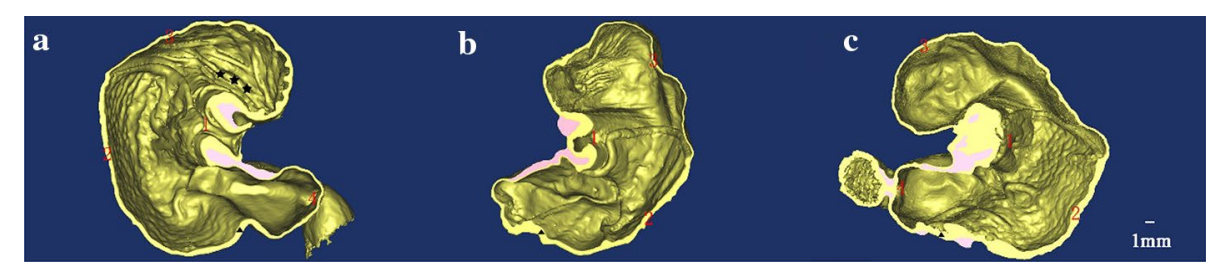

Fig. $63 \mathrm{D}$ anatomical structure images of the coronal plane of different stages of the stomach. a 3D internal structure image of the 4-week-old rat stomach. The stomach is round with obvious wrinkles in the fundus part, and there is a clear demarcation line between the gastric fundus and gastric corpus. b 3-dimensional internal structure image of the 6-week-old rat stomach. The wrinkles of the fundus part are decreasing, and the gastric corpus is becoming rugged. $\mathbf{c} 3$-dimensional internal structure image of the 12-week-old rat stomach. The stomach is elliptic, and the fundus part of it has become smooth. $\star \star \star$ represents the wrinkles of the gastric fundus. $\boldsymbol{\Delta}$ is the same size in the $\mathbf{a}, \mathbf{b}$ and $\mathbf{c}$ part of Fig. 6 and represents the thickness of the wall of the gastric corpus. 1 Cardia, 2 Gastric Corpus, 3 Gastric Fundus, 4 Pylorus
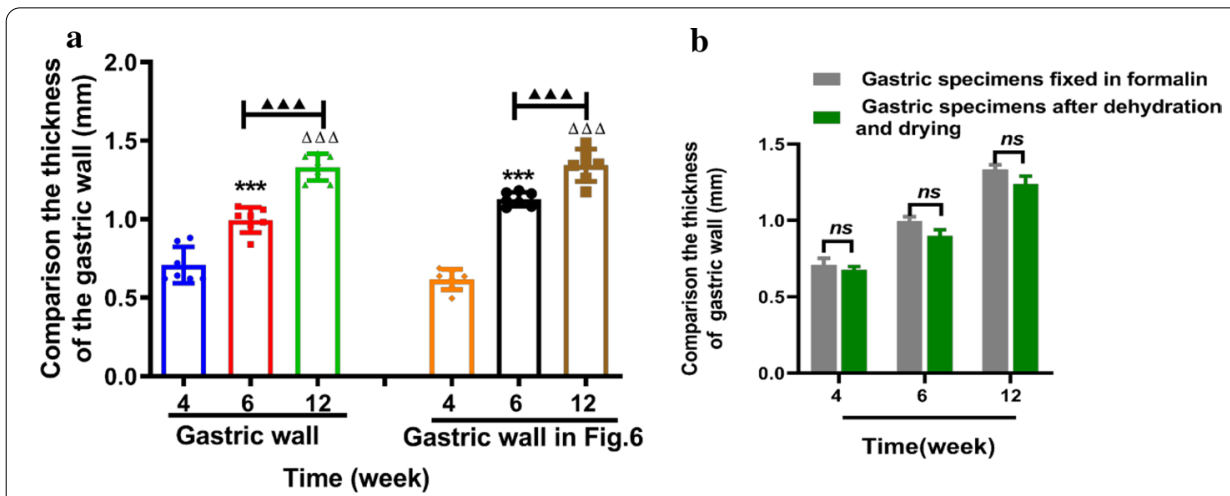

Fig. 7 The comparison results of the thickness of the gastric wall at various stages between gastric specimens and 3D figures in Fig. 6 and gastric specimens after dehydration and drying. a The thickness of the gastric wall of the specimens is significant increased from $0.71 \pm 0.04 \mathrm{~mm}$ to $0.99 \pm 0.05 \mathrm{~mm}, 1.33 \pm 0.32 \mathrm{~mm}$ about $28.28 \%(p<0.001, n=7), 46.61 \%(p<0.001, n=7)$ in the 4 weeks, 6 weeks and 12 weeks postnatal, respectively. And the thickness of gastric walls was calculated as shown in Fig. 6, with a marked enhancement from $0.62 \pm 0.024 \mathrm{~mm}$ to $1.13 \pm 0.02 \mathrm{~mm}, 1.34 \pm 0.04 \mathrm{~mm}$ about $45.13 \%(p<0.001, n=7)$ and $53.73 \%$ $(p<0.001, n=7)$. All the data at the same stage have no difference. Data represent mean \pm S.E.M. . $^{* * *} P<0.001$, $P<0.001, \mathbf{\Delta} \mathbf{\Delta} \mathbf{\Delta}_{P<0.001)}{ }^{* * *}$ 4-week-old vs 6-week-old, 4-week-old vs 12-week-old, $\mathbf{\Delta} \mathbf{\Delta} \mathbf{\Delta} 6$-week-old vs 12-week-old. $\mathbf{b}$ The thickness of gastric wall tends to decrease from $0.71 \pm 0.044$ to $0.62 \pm 0.05 \mathrm{~mm}$ by $4.6 \%$ $(p>0.05, n=7)$ in the 4 weeks models, from $1.00 \pm 0.05$ to $0.90 \pm 0.04 \mathrm{~mm}$ by $9.6 \%(p>0.05, n=7)$ in the 6 weeks models, and from $1.33 \pm 0.08$ to $1.210 \pm 0.06 \mathrm{~mm}$ by $9.12 \%(p>0.05, n=7)$ in the 12 weeks models, respectively. These results showed that the gastric shape and structure unchanged, and the thickness of gastric wall decreased proportionally less than $10 \%$ within the credible range of no difference

\section{Discussion}

Limitations of XILPCI must be overcome if XILPCI is to have wider applications. One limitation is that it takes around $1 \mathrm{~h}$ to shoot a larger specimen as it requires images shooting in several segments. The experiment is only effective for static specimens, so the device must be improved to shorten the imaging time required for living specimens. In addition, movement artifacts will appear when the specimens being taken are moving. In this experiment, because of the high resolution of the imaging, a merely small movement can produce imaging artifacts. Specimens shrinkage occurs when the specimens are taken on the sample table, which also produces movement artifacts under X-ray irradiation. The current solution is to make the specimen as dry 
as possible so as to shorten the experiment time. Movement artifacts can also affect the image quality and require further solution in later researches.

The CCD camera of BL13W1 of SSRF can obtain a resolution of $0.37 \mu \mathrm{m}$, making the XILPCI helpful for angiography. At present, Applications on humans in the field of phase-contrast mammography have been reported using both synchrotron radiation and conventional sources [9]. With further development, XILPCI would be a valuable imaging method for medical researches.

\section{Conclusion}

In summary, we have applied the XILPCI method to imaging of the rat stomach without the need for imaging agents. The XILPCI projection images showed that the development of the normal gastric structure may cause thickening of the gastric wall and coarseness of the gastric texture.

XILPCI is an imaging method using the X-ray phase variation. This makes it able to achieve micron-scale image resolution of biological tissue and can probably replace the traditional X-ray absorption method with synchrotron radiation sources. Due to the advantages mentioned above, the XILPCI method is likely to be widely used in the future at a low cost.

\section{Methods}

\section{Setup and specimens}

Twenty-one Sprague-Dawley healthy male rats were purchased from the Animal Centre of Capital Medical University in China. The rats were randomly divided into three groups with seven rats in each one. The rats were housed in cages under a controlled temperature of $22.0 \pm 1.0^{\circ} \mathrm{C}$ and 12 -h light-dark cycles. They were fed on standard laboratory chow and water and allowed to acclimate for more than 7 days. The rats were starved for $12 \mathrm{~h}$ and killed under anesthesia with an intraperitoneal injection of pentobarbital sodium at 4, 6 and 12 weeks of age. The stomach of the rat was removed and the stomach cavity is filled with $10 \%$ formalin to maintain the morphological structure of the stomach for X-ray image scanning. Stomach specimens were shown Fig. 8. These stomach tissues were cleaned with normal saline, fixed in $10 \%$ neutral phosphate-buffered formalin solution for $24 \mathrm{~h}$ and embedded in paraffin. $5 \mu \mathrm{m}$ thick of the specimens were obtained from the stomach and stained with HE staining. This animal study was in strict accordance with the recommendations in the Guide for the Care and Use of Laboratory Animals of the National Institutes of Health. The animal welfare Committee on the Ethics of Animal Experiments of Capital Medical University (Protocol No. AEEI-2016-079) approved the experiment protocol. All surgeries were performed under pentobarbital sodium anesthesia, and all efforts were made to minimize suffering.

\section{Imaging principle of XILPCI}

XILPCI experiments were done at synchrotron radiation [10] facility. Synchrotron radiation is electromagnetic radiation which is emitted by relativistic charged particles traveling along a turning orbit under the action of electromagnetic field. Synchrotron radiation, as a light source, has an obvious high-brilliance and flux, wide energy spectrum and very short pulses. 


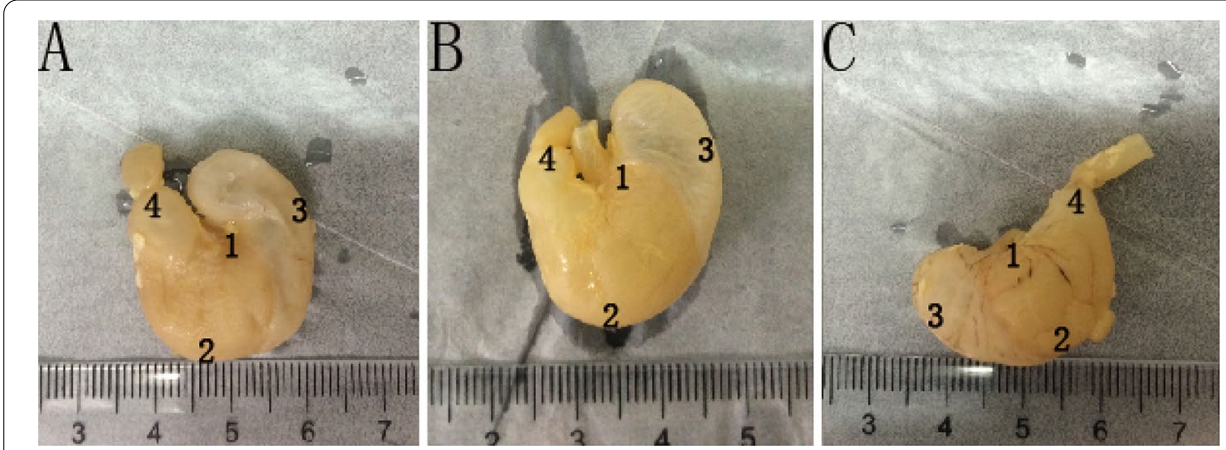

Fig. 8 Rats' gastric specimens. A A 4-week-old gastric specimen. B A 6-week-old gastric specimen. C A 12-week-old gastric specimen. 1 Cardia, 2 Gastric Corpus, 3 Gastric Fundus, 4 Pylorus. All the positions have been noted on the gross anatomy

The gastric XILPCI experiments were performed using the BL13W1 beamline of the Shanghai Synchrotron Radiation Facility (SSRF). The BL13W1 beamline partial facility of SSRF was depicted as shown in Fig. 9. A. Snigirev [5,11-13] obtained phase-contrast images using a synchrotron radiation light in 1995. The XILPCI method can use multicolor light sources, therefore, eliminate the need for the burdensome complexity of a monochrome system.

The complex refractive index $\mathrm{n}$ can be used to depict the XILPCI characteristics. The refractive index $\mathrm{n}$ is smaller than the number 1 and $\mathrm{n}$ formula is shown as follows:

$$
n=1-\delta-i \beta
$$

After X-rays go through an object, their phase and amplitude change. Real component presents the phase changes, while imaginary part presents the amplitude attenuation. In XILPCI of lighter elements (C, H, O, etc.) of the object, $\delta$ is 1000 times greater than $\beta$,

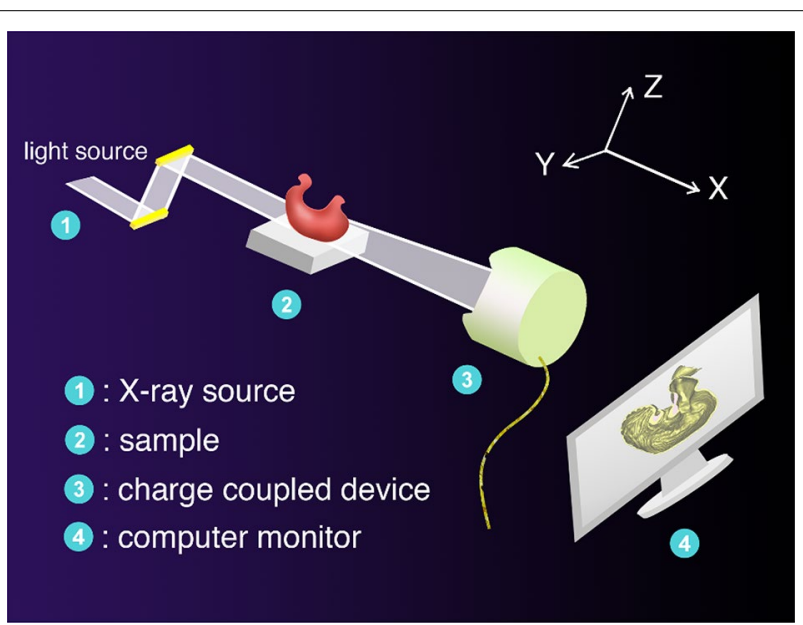

Fig. 9 Schematic diagram of BL13W1 beam line of SSRF. (1) The light source is used for the calibration location of the light, specimen and the CCD. (2) A multidimensional sample table. The specimens are placed on the sample table to rotate and their images are then obtained at different angles. (3) An X-ray CCD. It obtains specimens' projection images with high-resolution. (4) Data processor. It could calculate optical density from the CCD to the specimens and image conversion 
so the phase-change quantity is much larger than the change quantity of X-ray absorption attenuation. There is a phase-contrast imaging of micron-scale image resolution, so XILPCI images can show microstructures of objects.

\section{Steps of XILPCI}

Specific experimental methods: first, to reduce the XILPCI artifacts caused by specimen deformation, the specimens are placed in the air and dried for a period of time. Then the dry specimens were wrapped with insulating materials and placed on the sample table.

After evaluating different levels of X-ray energy, we chose the energy level of $17.5 \mathrm{keV}$ to be used for this experiment. The energy value can be changed and is determined according to the specimen size. Different specimens have different energy values to obtain clear X-ray phase-contrast images. The images were whiter if the energy was higher than $17.5 \mathrm{keV}$, and the imaging exposure time also increased when the energy was lower than $17.5 \mathrm{keV}$. Images will be dark if the exposure time is not appropriate. It will also take a longer time to shoot those images required for $\mathrm{CT}$ if the exposure time increases and gastric specimens will undergo serious deformation. Therefore, $17.5 \mathrm{keV}$ is an optimal parameter of comprehensive factors. The distance from the light source to the specimen was $59.3 \mathrm{~m}$ and the detector was $60 \mathrm{~cm}$ from the specimen to CCD, with 9- $\mu \mathrm{m}$ image resolution and an exposure time of $8 \mathrm{~ms}$. It took more $20 \mathrm{~min}$ to obtain XILPCI projection images of a gastric specimen using 0.1 degree steps from 0 to 180 degrees over the gastric specimen.

\section{Gastric wall thickness measurement}

To further compare the effects of stomach drying and atrophy caused by exposure to air under the test conditions on the structure, the specimens fixed with $10 \%$ formalin were exposed to air at the same temperature and for the same duration in this experiment. After 20 min of drying, the thickness of the gastric wall was measured under stereoscope with the vernier caliper. Three times for each position, seven arbitrary parts of gastric walls of all of dry specimens were selected and the average value was taken. The value is expressed as Mean \pm SEM.

Pre-dehydration wall thickness measurement was the same conditions with the dehydration wall thickness. The stomach was taken out from $10 \%$ formalin solution and kept moist. The gastric walls thickness was measured at seven arbitrary parts of all of moist specimens, three times for each position, under the same stereoscope with the same vernier caliper. For further statistical significance, all gastric walls were randomly selected. The value is also expressed as Mean \pm SEM.

\section{Histology and pathology scoring}

Stomach tissue specimens were dewaxed and stained with the HE staining. Sections were fixed on microscope slides and observed with an Olympus DP72 MacroView (Japan). Histological scoring was based on a previously adapted scoring system. 


\section{Statistical analysis}

The data were analyzed using GraphPad Prism 5.0 software package (GraphPad Software Inc., San Diego, CA, USA) of variance of the gastric wall thickness and all values were expressed as mean and standard error of mean (S.E.M.); $n$ was the number of animal in each experiment. The differences among groups were analyzed using a one-way analysis of variance followed by Dunnett's multiple comparison. A $p$ value less than 0.05 was considered statistically significant. The power of the results was $86.5 \%$ by the software power analysis and specimen size software.

\section{Abbreviations}

XILPCI: X-ray in-line phase-contrast imaging; CCD: Charge coupled device; BL13W1: Imaging and biomedical application beamline; SSRF: Shanghai Synchrotron Radiation Facility; 3D: Three dimensional.

\section{Supplementary Information}

The online version contains supplementary material available at https://doi.org/10.1186/s12938-021-00866-8.

Additional file 1: Video S1. 3D video of a 4-week-old rat stomach.

Acknowledgements

Our deeply gratitude goes the assistance of all the staff in BL13W1 from Shanghai Synchrotron Radiation Facility.

Authors' contributions

QT completed the experimental data, worked on the algorithm design, measured the thickness of gastric wall and drafted the manuscript. JDX conceived the study, contributed to specimen preparation and conclusions and manuscript modification. XHT and CCG contributed to the animal handling. SZY reconstructed the 3-dimensional reconstruction images. All the authors read and approved the final manuscript.

\section{Funding}

This study was supported by the National Natural Science Foundation of China (Grant No. 81673671 and 81274173); the Natural Science Foundation of Beijing (Grant No. 7144189 and 7122017); and the Science Foundation of Capital Medical University (Grant No. 17ZR24).

Availability of data and materials

The datasets generated during the current study are available from the corresponding author on reasonable request.

\section{Declarations}

Ethics approval and consent to participate

Date of our animal study trial registration: 2015-07-11. Registered with the Guide for the Care and Use of Laboratory Animals of the National Institutes of Health. (Protocol No. AEEl-2016-079).

\section{Consent for publication}

All the authors consent for publication.

\section{Competing interests}

The authors declare no competing interests.

\section{Author details}

${ }^{1}$ School of Biomedical Engineering, Capital Medical University, Beijing 100069, China. ${ }^{2}$ Department of Physiology and Pathophysiology, School of Basic Medicine, Capital Medical University, Beijing 100069, China. ${ }^{3}$ Experimental Centre for Basic Medical Teaching, School of Basic Medicine, Capital Medical University, Beijing 100069, China.

Received: 29 June 2020 Accepted: 9 March 2021

Published online: 20 March 2021

\section{References}

1. McGrath PS, Wells JM. SnapShot: Gl tract development. Cell. 2015;161(1):176-176e171.

2. Majumdar AP, Johnson LR. Gastric mucosal cell proliferation during development in rats and effects of pentagastrin. Am J Physiol. 1982;242(2):G135-139.

3. Stoddart RW, Widdowson EM. Changes in the organs of pigs in response to feeding for the first $24 \mathrm{~h}$ after birth. III. Fluorescence histochemistry of the carbohydrates of the intestine. Biol Neonate. 1976;29(1-2):18-27. 
4. Moon HW, Joel DD. Epithelial cell migration in the small intestine of sheep and calves. Am J Vet Res. 1975;36(2):187-9.

5. Snigirev A, Snigireva I, Kohn V, Kuznetsov S, Schelokov I. On the possibilities of $x$-ray phase contrast microimaging by coherent high-energy synchrotron radiation. Rev Scientific Instruments. 1995;66(12):6.

6. Gluck N, Shpak B, Brun R, Rosch T, Arber N, Moshkowitz M. A novel prepless X-ray imaging capsule for colon cancer screening. Gut. 2016;65(3):371-3.

7. Kimura H, Momiyama M, Tomita K, Tsuchiya H, Hoffman RM. Long-working-distance fluorescence microscope with high-numerical-aperture objectives for variable-magnification imaging in live mice from macro- to subcellular. J Biomed Opt. 2010;15(6):066029.

8. Qiang T, Luo S. The research of cancer imaging by X-ray in-line phase-contrast imaging technique. Adv Materials Res. 2013;718:3.

9. Bravin A, Coan P, Suortti P. X-ray phase-contrast imaging: from pre-clinical applications towards clinics. Phys Med Biol. 2013:58(1):R1-35.

10. Schramm E, Muhlberger F, Mitschke S, Reichardt G, Schulte-Ladbeck R, Putz M, Zimmermann R. Determination of the ionization potentials of security-relevant substances with single photon ionization mass spectrometry using synchrotron radiation. Appl Spectrosc. 2008;62(2):238-47.

11. Ferrero C, Smilgies DM, Riekel C, Gatta G, Daly P. Extending the possibilities in phase space analysis of synchrotron radiation x-ray optics. Appl Opt. 2008;47(22):E116-124.

12. Rack A, Garcia-Moreno F, Schmitt C, Betz O, Cecilia A, Ershov A, RackT, Banhart J, Zabler S. On the possibilities of hard $X$-ray imaging with high spatio-temporal resolution using polychromatic synchrotron radiation. J Xray Sci Technol. 2010;18(4):429-41.

13. Billinghurst BE, Bergstrom JC, Baribeau C, Batten T, Dallin L, May TE, Vogt JM, Wurtz WA, Warnock R, Bizzozero DA, et al. Observation of wakefields and resonances in coherent synchrotron radiation. Phys Rev Lett. 2015;114(20):204801.

\section{Publisher's Note}

Springer Nature remains neutral with regard to jurisdictional claims in published maps and institutional affiliations.

- fast, convenient online submission

- thorough peer review by experienced researchers in your field

- rapid publication on acceptance

- support for research data, including large and complex data types

- gold Open Access which fosters wider collaboration and increased citations

- maximum visibility for your research: over $100 \mathrm{M}$ website views per year

At BMC, research is always in progress.

Learn more biomedcentral.com/submissions 